\title{
Efectos nutricionales de los polisacáridos no amiláceos en pollo de engorde de la línea Ross
}

\author{
Nutritional impact of non-starch polysaccharides in \\ broilers Ross line
}

Diana Patricia Alba Laverde ${ }^{1}$

\begin{abstract}
Resumen
Los Polisacáridos no Amiláceos (PNA) son un tipo de carbohidratos presentes en antinutricionales con el fin de disminuir los niveles de grasa corporal. Para esta investigación se contó con tres grupos de pollos de engorde de la línea Ross: al primero se le incluyó alcachofa (Cynara scolymus) en la dieta; al segundo, cebada (Hordeum vulgare), y al tercero se le dejó sólo el alimento balanceado comercial. Como resultado final se obtuvo un menor porcentaje de grasa en canal en los pollos del tratamiento 1, seguido por los pollos del tratamiento 2, y los mayores porcentajes de grasa en canal fueron los de los pollos del grupo 3 o control. Como resultado adicional, en este grupo 3 se presentaron muertes por Síndrome Ascítico (SA), con lo cual se plantea la posibilidad de emplear los PNA, con el fin de disminuir la presentación de enfermedades metabólicas.
\end{abstract}

Palabras clave: Grasa corporal, Polisacáridos, Ascitis. (Fuente: DeCS)

\begin{abstract}
The non-starch polysaccharides (NSP) are a type of carbohydrates present anti-nutritional factors to reduce body fat levels. In this research, It were used three groups of broilers Ross line for this research: In the first was included artichoke (Cynara scolymus) on diet; the second barley (Hordeum vulgare) and the third group commercial food. As a final result a minor percentage on carcass fat was obtained in the first group followed by the broilers in group two and the higher results of carcass fat was of the third group or control. As additional result in the third group was reported 3 deaths due ascites syndrome, which raises the possibility of using NSP to decrease the occurrence of metabolic diseases.
\end{abstract}

Key words: Body fat, Polysaccharides, Ascites. (Source: DeCS)

Médico Veterinario Zootecnista. Universidad Pedagógica y Tecnológica de Colombia. Tunja,

Colombia. dalba359@hotmail.com 


\section{Introducción}

En los últimos años se ha incrementado la preocupación de la sociedad mundial por consumir alimentos con la menor cantidad de factores de riesgo para la salud. El engrasamiento del pollo es una de las características de las líneas genéticas actualmente utilizadas (Crespo, 2004), además, produce efectos económicos y sociales indeseables, ya que deteriora la conversión alimenticia y disminuye el rendimiento de canal, debido a la eliminación de la grasa abdominal en el momento del beneficio (Aránibar y Mateos, 2002).

El desarrollo que se ha dado en la producción avícola es, básicamente, debido a la preocupación por una mejor y balanceada nutrición (Hans y Aguirre, 2002). Dentro de los contenidos nutricionales de algunos alimentos se encuentran los denominados carbohidratos clasificados como Polisacáridos No Amiláceos (PNA), que son considerados por muchos investigadores como sustancias antinutricionales (Mateos et al., 2006), debido a las repercusiones que se presentan en la capacidad de intercambio catiónico y de hidratación, y a su viscosidad y su habilidad para absorber compuestos orgánicos, ya que tienen la propiedad de pegarse a los alimentos y no dejarlos absorber a nivel intestinal (Escobar, 1998). No obstante, también se reporta que inclusiones bajas de PNA en la alimentación animal trae beneficios como disminución del colesterol sérico, efectos antibacteriales y producción de carnes con contenidos bajos en grasas (Francesh, 1996).

Partiendo de este hecho, se planteó realizar inclusiones bajas de cebada (Hordeum vulgare) y alcachofa (Cynara scolymus), alimentos ricos en PNA, en la dieta de pollos de engorde, con el objetivo principal de evaluar su efecto sobre los niveles de producción de grasa abdominal, específicamente ganancia de peso, conversión de alimento y rendimiento de los pollos en canal, estableciendo así los efectos presentes sobre la productividad y eficiencia de los animales; adicional a lo anterior, se definieron las enfermedades que se presentaron durante el desarrollo de la investigación, y, finalmente, se evaluaron y compararon los pesos en canales, vísceras y grasa abdominal.

\section{Materiales y Métodos}

La investigación se realizó en las instalaciones de la Clínica Veterinaria de la Universidad Pedagógica y
Tecnológica de Colombia, en la ciudad de Tunja, situada en el centro oriente de Colombia, a una altura sobre el nivel del mar de $2.775 \mathrm{~m}$, con una temperatura promedio de 12 a $14{ }^{\circ} \mathrm{C}$, una precipitación promedio de $750 \mathrm{~mm}$ por año, y una humedad relativa del $70 \%$ al $80 \%$.

Esta fue una investigación de tipo experimental, en la cual se probaron los resultados de la inclusión en la dieta para pollos de engorde (un alimento balanceado comercial) de un $5 \%$ de cebada (Hordeum vulgare) o de alcachofa (Cynara scolymus), dos alimentos ricos en PNA.

Se emplearon para la investigación 60 pollos de la línea Ross x Ross, de un día de nacidos, los cuales se dividieron en tres grupos, cada uno de 20 pollos seleccionados completamente al azar; los grupos se denominaron de la siguiente manera:

- Tratamiento 1: alimento balanceado comercial, más el 5\% de harina de alcachofa (Cynara scolymus).

- Tratamiento 2: alimento balanceado comercial, más $5 \%$ de harina de cebada (Hordeum vulgare).

- Control: únicamente con alimento balanceado comercial.

Se manejaron registros de los pesajes semanales de los pollos, de la cantidad de alimento administrada y de las situaciones anormales que se presentaban. Durante la cuarta semana se presentaron problemas de tipo respiratorio, que se manifestaron con estornudos y secreción nasal y ocular; un pollo del control murió tras presentar postración, plumas erizadas y dificultad respiratoria. Finalmente, en la quinta semana murieron otros dos pollos, pertenecientes al control. En la necropsia se encontró: cúmulo de abundante líquido y fibrina en abdomen e hígado, pulmones e hígado notablemente congestionados, bordes hígado redondeados y corazón agrandado. Relacionando los síntomas presentados y los hallazgos en necropsia, se diagnosticó Síndrome Ascítico (SA), razón por la cual se decide someter los pollos a un ayuno de 24 horas y restringir la cantidad de alimento administrada.

Para el análisis estadístico de variables productivas, además de las características de las canales, se empleó un diseño completamente al azar, un análisis por ANOVA y se aplicó el test de Tukey, el cual tiene un $95 \%$ de confiabilidad. A partir de la cuarta semana, debido a la reducción de la 
muestra en los tratamientos 1 y 2, el programa utiliza la media armónica para el análisis de los datos.

\section{Resultados y Discusión}

Consumo de alimento, ganancia de peso y conversión alimenticia. Al analizar los resultados de los pesajes realizados semanalmente, no arrojaron diferencias estadísticamente significativas entre los tratamientos y el control, sin embargo, la ganancia de peso mayor fue la obtenida por el control, seguida por el tratamiento 2, y más baja la del tratamiento 1 .

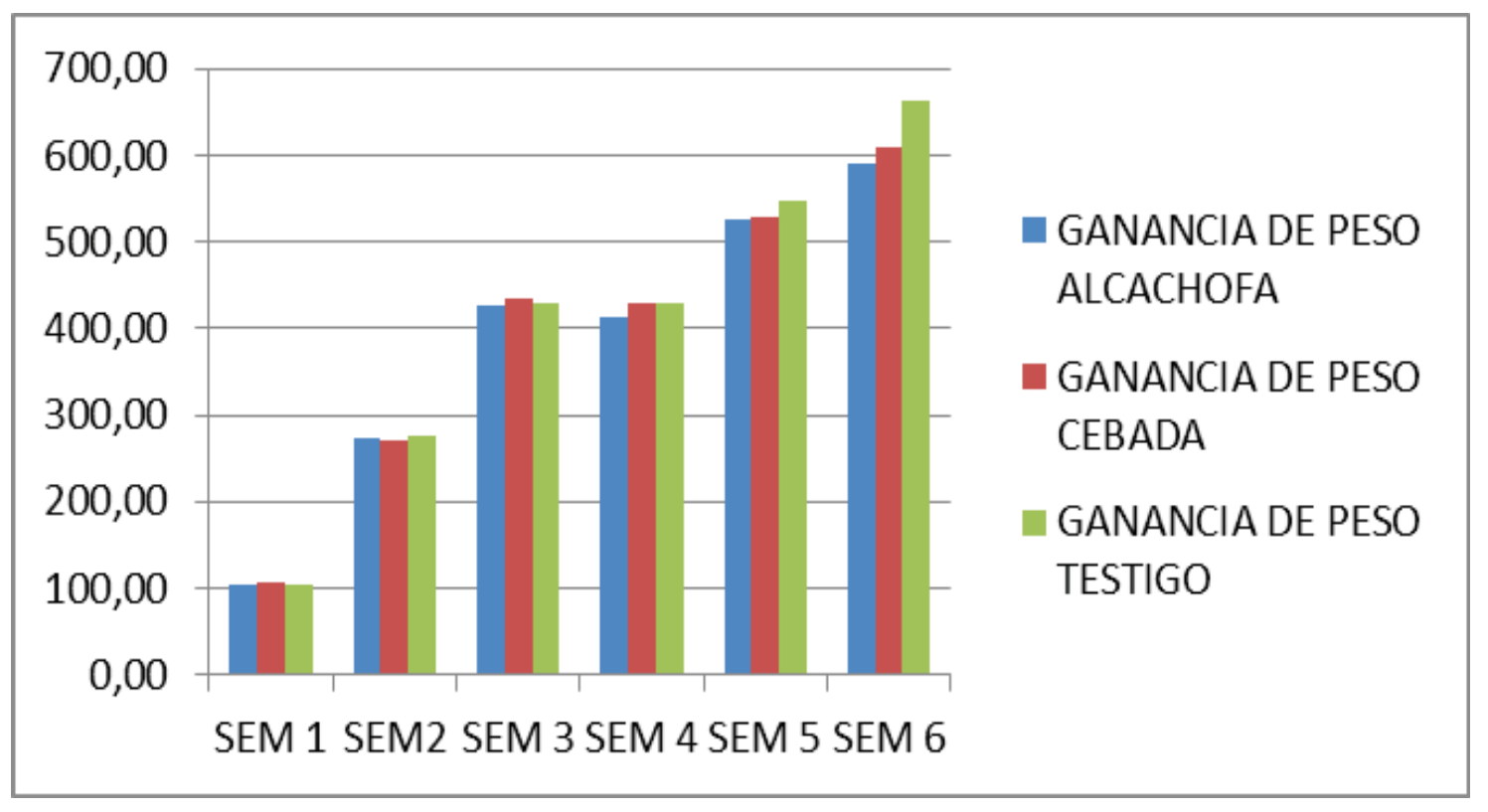

Figura 1. Ganancia de peso semanal

Varios autores plantean que la inclusión de PNA en dietas para pollos de engorde afecta negativamente los parámetros productivos, efecto corroborado en esta investigación, siendo más evidente en el tratamiento 1 , en el que durante las 6 semanas se observó una ganancia de peso inferior. El consumo de PNA (fibra alimentaria) en diferentes cantidades promueve la pérdida de peso (OMS, 2003), debido, en los pollos, a que se reduce la digestibilidad mediante el simple encapsulamiento del material contenido dentro del endospermo; es así como las células intactas del endospermo son resistentes a la digestión, y así resulta la reducción de digestibilidad (Escobar, 1998).

De hecho, trabajos publicados establecieron que, en pollos, la fibra no contribuía al valor alimenticio del pienso, tras encontrar una fuerte correlación negativa entre el contenido de fibra y la digestibilidad de proteína, de grasa e hidratos de carbono (García, 2000), llegando a recomendar utilizar la fibra para predecir el valor nutritivo de los alimentos (Mateos et al, 2006).

Los resultados obtenidos corroboran las afirmaciones hechas acerca de los efectos de la inclusión de PNA, además de los producidos por la restricción alimenticia, en donde el crecimiento del pollo es proporcional a la reducción del consumo de alimento (González et al., 2000).

Tabla 1. Ganancia de peso semanal

\begin{tabular}{|c|c|c|c|}
\hline SEMANA & TRATAMIENTO 1 & TRATAMIENTO 2 & CONTROL \\
\hline $\mathbf{1}$ & $104,92 \mathrm{a}$ & $107,04 \mathrm{a}$ & $103,05 \mathrm{a}$ \\
\hline $\mathbf{2}$ & $274,00 \mathrm{a}$ & $271,15 \mathrm{a}$ & $276,15 \mathrm{a}$ \\
\hline $\mathbf{3}$ & $426,05 \mathrm{a}$ & $434,25 \mathrm{a}$ & $429,75 \mathrm{a}$ \\
\hline $\mathbf{4}$ & $413,42 \mathrm{a}$ & $427,75 \mathrm{a}$ & $430,00 \mathrm{a}$ \\
\hline $\mathbf{5}$ & $526,11 \mathrm{a}$ & $527,89 \mathrm{a}$ & $548,00 \mathrm{a}$ \\
\hline $\mathbf{6}$ & $590,56 \mathrm{a}$ & $608,42 \mathrm{a}$ & $644,00 \mathrm{a}$ \\
\hline
\end{tabular}


El consumo de alimento semanal fue el indicado por la guía de manejo de pollo de engorde

Ross; a partir de la cuarta semana se administró de manera restringida, con el fin de controlar y evitar un brote de Síndrome Ascítico (SA); la restricción alimenticia es una práctica de uso normal para reducir la incidencia de enfermedades metabólicas como la muerte súbita y la ascitis (Arce, 1993; López, 2001, y Suárez et al., 2003), aunque esta restricción limitó un poco la ganancia de peso, aumentó nuevamente en las semanas 5 y 6 .

Tabla 2. Resultados del pesaje semanal en gramos

\begin{tabular}{|c|c|c|c|}
\hline SEMANA & $\begin{array}{c}\text { TRATAMIENTO } \\
\mathbf{1}\end{array}$ & $\begin{array}{c}\text { TRATAMIENTO } \\
\mathbf{2}\end{array}$ & CONTROL \\
\hline $\mathbf{1}$ & 144,50 & 146,35 & 142,35 \\
\hline $\mathbf{2}$ & 418,50 & 417,50 & 423,25 \\
\hline $\mathbf{3}$ & 844,47 & 851,75 & 853,00 \\
\hline $\mathbf{4}$ & 1257,89 & 1279,50 & 1283,00 \\
\hline $\mathbf{5}$ & 1781,67 & 1807,37 & 1831,00 \\
\hline $\mathbf{6}$ & 2372,22 & 2415,79 & 2495,00 \\
\hline
\end{tabular}

El índice de conversión alimenticia presentó variaciones en los tres tratamientos durante las 6 semanas; sin embargo, a partir de la segunda semana (momento en que se inicia la inclusión de PNA) hasta la sexta semana, el índice menor fue el obtenido por el control, seguido, en orden ascendente, por el del tratamiento $2 \mathrm{y}$, finalmente, por el tratamiento 1 , que resultó ser el mayor.
Estos resultados indican que el pollo es más eficiente con el alimento balanceado comercial, seguido por la inclusión del $5 \%$ de cebada (Hordeum vulgare), y en menor medida con la inclusión del 5\% de alcachofa (Cynara scolymus). Mientras menor sea el índice de conversión alimenticia, más eficiente es el pollo; los resultados obtenidos indican que la inclusión de PNA en dietas para pollos de engorde hace menos eficiente el rendimiento de éstos.

Tabla 3. Conversión alimenticia

\begin{tabular}{|c|c|c|c|}
\hline SEMANA & $\begin{array}{c}\text { TRATAMIENTO } \\
\mathbf{1}\end{array}$ & $\begin{array}{c}\text { TRATAMIENTO } \\
\mathbf{2}\end{array}$ & CONTROL \\
\hline $\mathbf{1}$ & $1,12 \mathrm{a}$ & $1,11 \mathrm{a}$ & $1,13 \mathrm{a}$ \\
\hline $\mathbf{2}$ & $1,25 \mathrm{a}$ & $1,26 \mathrm{a}$ & $1,24 \mathrm{a}$ \\
\hline $\mathbf{3}$ & $1,36 \mathrm{a}$ & $1,35 \mathrm{ab}$ & $1,35 \mathrm{~b}$ \\
\hline $\mathbf{4}$ & $1,64 \mathrm{a}$ & $1,62 \mathrm{a}$ & $1,61 \mathrm{a}$ \\
\hline $\mathbf{5}$ & $1,82 \mathrm{a}$ & $1,80 \mathrm{a}$ & $1,77 \mathrm{a}$ \\
\hline $\mathbf{6}$ & $1,96 \mathrm{a}$ & $1,93 \mathrm{ab}$ & $1,87 \mathrm{~b}$ \\
\hline
\end{tabular}

Letras diferentes en la misma fila significan diferencias estadísticamente significativas $(p<0,05)$.

En los resultados de conversión alimenticia, en la tercera y sexta semana se presentaron diferencias estadísticamente significativas entre el tratamiento 1 y el control; sin embargo, entre éstos y el tratamiento 2 no se presentaron.

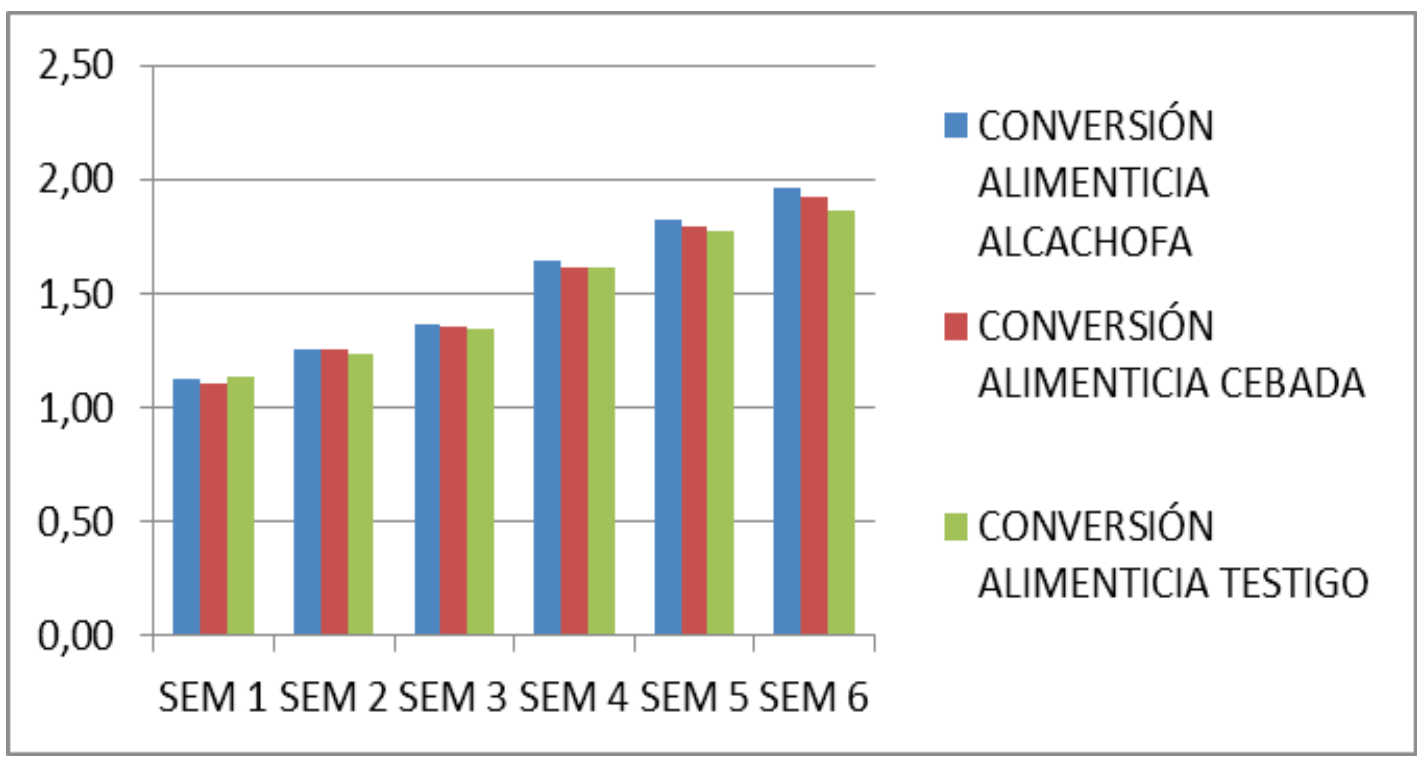

Figura 2. Conversión alimenticia 
Enfermedades presentes en el estudio. Terminando la segunda semana de edad, momento en que se dio inicio a la inclusión del $5 \%$ de alcachofa (Cynara scolymus), para el tratamiento 1, y 5\% de cebada (Hordeum vulgare), para el tratamiento 2 , las aves presentaron diarrea, efecto producido por la inclusión de PNA en sus dietas a edad temprana (Choque, 2008), debido a que el tránsito de fibra en grandes cantidades por el tracto gastrointestinal (TGI) predispone a la colonización de bacterias perjudiciales para éste; sin embargo, estos niveles de fibra en el pollo adulto tienen menores posibilidades de producir enfermedades gastrointestinales, debido al fortalecimiento del sistema digestivo durante su desarrollo; es así como en las semanas posteriores no presentaron problemas digestivos.

Entre la tercera y cuarta semana se presentó una afección respiratoria que se manifestó con secreciones oculares y nasales, además de estornudos, y posterior a esto murieron tres pollos con síntomas como postración, plumas erizadas y dificultad respiratoria.

El rápido crecimiento de las líneas de pollo de engorde empleadas actualmente, en las cuales el desarrollo músculo-esquelético no es proporcional al de pulmones y corazón (López, 1991), y las alteraciones en el aparato respiratorio, debido, entre otras razones, a la crianza de aves en alturas elevadas (más de los $2.250 \mathrm{msnm}$ ), en donde la presión parcial de oxígeno es menor, son factores predisponentes a la presentación de SA (Cortez et al., 2006).
Al realizar la necropsia se encontró líquido con fibrina en toda la cavidad abdominal, los bordes del hígado redondeados, corazón agrandado y congestión pulmonar y hepática, signos característicos del SA (Arce et al., 1991).

En el momento en que se presentaron las muertes se realizó la restricción alimenticia durante 24 horas, y en los 4 días posteriores, ayunos de 5 horas, aproximadamente. La restricción alimenticia, la falta de acceso al alimento y la disminución en el valor nutricional de este son prácticas comunes para el control del SA.

Así como la restricción alimenticia es una práctica común para controlar o disminuir la presentación de SA, también lo es la administración de alimento cuyo contenido de proteína y grasa sean deficientes (factores cuya absorción limitan los PNA).

Lo anterior, sumado al hecho de que los pollos que murieron pertenecían al control, expone la posibilidad de que la inclusión de los PNA en dietas para pollos de engorde disminuya la presentación de SA, debido a la menor ganancia de peso y a una menor cantidad de grasa corporal.

Pesos en canal, vísceras y grasa abdominal. $\mathrm{Al}$ cumplir los 45 días de edad fueron sacrificados 24 pollos, que primero fueron pesados completos, y luego, separando de la canal, vísceras (incluyendo patas y cabeza) y grasa abdominal.

Tabla 4. Pesos en canal

\begin{tabular}{|l|c|c|c|c|}
\hline TRATAMIENTO & $\begin{array}{c}\text { PESO DE CANAL } \\
\text { COMPLETA (gr) }\end{array}$ & $\begin{array}{c}\text { PESO DE } \\
\text { CANAL (gr) }\end{array}$ & $\begin{array}{c}\text { PESO DE } \\
\text { VísCERAS (gr) }\end{array}$ & $\begin{array}{c}\text { PESO DE GRASA } \\
\text { ABDOMINAL (gr) }\end{array}$ \\
\hline Alcachofa & $1422,75 \mathrm{a}$ & $1137,5 \mathrm{a}$ & $247,8 \mathrm{a}$ & $37,375 \mathrm{a}$ \\
\hline Cebada & $1493,78 \mathrm{a}$ & $1200 \mathrm{a}$ & $247,87 \mathrm{a}$ & $48,78 \mathrm{a}$ \\
\hline Control & $1452,5 \mathrm{a}$ & $1170,5 \mathrm{a}$ & $248 \mathrm{a}$ & $50,2 \mathrm{a}$ \\
\hline
\end{tabular}

Los pesos finales obtenidos por el tratamiento 1 fueron menores que los obtenidos por el tratamiento 2 y por el control, con lo cual se concluye que realizar inclusiones del $5 \%$ de alcachofa (Cynara scolymus) disminuye los pesos de las canales; sin embargo, la cantidad de grasa corporal es menor, lo cual es un punto a favor en la producción de carne de pollo. Es así como se confirma la influencia de la dieta sobre la cantidad de grasa acumulada por el pollo en su madurez (García, 2000; García et al., 2007). 
Los pesos finales obtenidos por el tratamiento 2 , en canales completas y sin vísceras, fueron los mayores; sin embargo, los pesos de vísceras (incluidas patas y cabezas) fueron menores que los obtenidos por el control, al igual que la cantidad de grasa corporal.

El análisis estadístico no arrojó diferencias significativas entre los pesos finales; no obstante, es importante destacar que la cantidad de grasa de cavidad abdominal del tratamiento 2 es muy similar a la obtenida por el control.

\section{Conclusiones}

La inclusión de alcachofa (Cynara scolymus) en dietas de pollo de engorde causa un menor rendimiento en los parámetros productivos, haciéndolos menos eficientes; igual sucede con la

\section{Literatura citada}

Araníbar, M., Mateos, G. G. (2002). Reduciendo el contenido de grasa en la canal del pollo de engorda. Portal avícola, porcícola y alimentos balanceados. Recuperado de http//www.midiatecavipec.com/avicultura/ avicultura230206 2.htm.

Arce, M. J. (1993). Restricción de alimento manual y diferentes densidades de nutrientes en las dietas para el control del síndrome ascítico en el pollo de engorda. XI Ciclo de Conferencias Internacionales sobre Avicultura. INIFAP$S A R H$. Centro de ganadería. Colegio de Postgraduados. Montecillo, Estado de México, México. 37-54.

Choque, J. A. (2008). Evaluación del estado oxidativo y salud intestinal de pollos de carne en respuesta a la alimentación con grasas recicladas. Tesis doctoral. Bellaterra. En http// www.tesisenxarxa.net/TESIS UAB/AVAILABLE/TDX-0925109-121137// jachl1de1.pdf.7p.

Cortés, A., Estrada, A., Ávila, E. (2006). Productividad y mortalidad por síndrome ascítico en pollos de engorde alimentados con dietas granuladas o en harina. Instituto Nacional de Investigaciones Forestales, Agrícolas y Pecuarias. Técnica Pecuaria de México, 44(2), 241-242.

Crespo, N. (2004). Reducción de la disposición de grasa abdominal en el pollo de carne inclusión de cebada (Hordeum vulgare), aunque en menor medida; resultados que son similares a los obtenidos con un alimento balanceado comercial. En etapas iniciales del desarrollo del pollo de engorde, la inclusión de alimentos ricos en polisacáridos no amiláceos es un factor predisponente para la colonización de bacterias en el tracto gastrointestinal, por lo que es frecuente la presentación de camas húmedas por diarreas; sin embargo, este tipo de alimentos ayudan a la maduración del sistema digestivo, haciendo al pollo adulto menos susceptible a padecerlas. EI rendimiento en canal de pollos a los que se les realizó la inclusión de polisacáridos no amiláceos en la dieta son menores a los obtenidos por pollos alimentados con alimento balanceado comercial, sin embargo, se obtiene un menor cúmulo de grasa, factor buscado por avicultores y consumidores.

mediante la modificación del perfil de ácidos grasos de la dieta. Recuperado de http://www. tesisenred.net/TDX-0704105-125918.

Escobar-Álvarez, M. V. (1998). Adición enzimática en dietas a base de maíz-soya para aves de postura comercial. Facultad de Medicina Veterinaria y Zootecnia de la Universidad de San Carlos de Guatemala. Guatemala. URL disponible en http://biblioteca.usac.edu.gt/ tesis/10/10_0733.pdf. [Fecha de acceso 20 de marzo de 2012]. 11 p.

Francesch, M. (1996). Bases de la utilización de complejos enzimáticos en avicultura. Instituto de Tecnologías Agroalimentarias. XII curso de especialización FEDNA. Madrid. URL disponible en http//www.etsia.upm.es/fedna/ capítulos/96capituloVIII.pdf. [Fecha de acceso 21 de marzo de 2012].

García, M. (2000). Evaluación de complejos enzimáticos en la alimentación de pollos de engorde. Universidad Politécnica de Madrid. Escuela Técnica Superior de Ingenieros Agrónomos: 4-5.

García, T. (2008). Industrialización integral de la alcachofa en pasta nutricional y para alimentos balanceados. Universidad Nacional Mayor de San Marcos. Industrial Data, 11(1), 2-6.

González, J., Suárez, M., Pro, A., López, C. (2000). Restricción alimenticia y salbutamol en el control del síndrome ascítico en pollos 
de engorda: 2. Respuesta hematológica y cardiaca. Agrociencia, 34(3), 294-299.

Hans, M., Aguirre, V. (2002). Avances en el mejoramiento de la producción avícola. Memorias del XI Congreso Venezolano de Producción e Industria Animal. ULA-Trujillo. Valera. URL disponible en http://avpa.ula.ve/ congresos/cd_xi_congreso/pdf/hansmann.pdf. [Fecha de acceso 18 de marzo de 2012].

López, C. (1991). Investigaciones sobre el síndrome ascítico en pollos de engorda. Universidad Nacional Autónoma de México. Facultad de Medicina Veterinaria y Zootecnia. Departamento de Producción Animal. México D. F. 17-18.
Mateos, G. G., Lázaro, R., González, J. M., Jiménez, E., Vicente, B. (2006). Efectos de la fibra dietética en piensos de iniciación para pollitos y lechones. Departamento de Producción Animal. Universidad Politécnica de Madrid. XXII curso de especialización FEDNA. Barcelona. $42 \mathrm{p}$.

Suárez, L., Fuentes, J., Torres, M., López, S. (2004). Efecto de la restricción alimenticia sobre el comportamiento productivo de pollos de engorda. Departamento de Ciencia Animal. Universidad Autónoma Antonio Narro. Revista Agraria Nueva Época 1(3), 24-30.

Fecha de Recepción: 8 de mayo de 2012

Fecha de Aceptación: 28 de abril de 2013 